\title{
A Diet ConTrol AND Fitness Assistant APPLICATION USING DEEP LEARNING-BASED IMAGE CLASSIFICATION
}

\author{
Tianren Dong ${ }^{1}$, Yu Sun ${ }^{2}$ and Fangyan Zhang ${ }^{3}$ \\ ${ }^{1}$ Northwood High School, Irvine, CA, 92620 \\ ${ }^{2}$ Department of Computer Science, California State Polytechnic University, \\ Pomona, CA, 91768 \\ ${ }^{3}$ ASML, San Jose, CA, 95131
}

\begin{abstract}
With more and more attentions paid on health, people begin to care about healthy diet options created by experts on nutrition. However, it will take a long time to observe the effects by taking healthy diet. This causes great difficulty for users to follow the healthy diet strictly. Most existing applications are not user-friendly in inputting information to the application. Then it becomes difficulty to track for exact health status. This paper proposes an android application which can be trained to recognize different kinds of food and facilitate the information input through phone camera using machine learning algorithms. Thus, nutritional information can be fed in application accurately.
\end{abstract}

\section{KEYWORDS}

Machine learning, Android application, Image recognition

\section{INTRODUCTION}

With such a large variety of healthy diet options proposed by either nutrition experts or fitness models, people are convinced to adopt a diet that promises them fascinating benefits and huge transformations [1][2]. However, it is hard for people to find the diet that best fits them out of all the promising choices despite the difficulty of strictly following their chosen diet [3][4]. There have been many mobile applications that provide a way for people to track their calories, nutrition, and exercise in order for them to keep up with their diet, but these applications have complicated food entry process [5][6][7]. APPNAME is an android application that helps people keep track of their consumed food and recognize their unhealthy eating habits. It uses a machine learning image recognition trained to recognize different kinds of food, making each food entry much simpler through recognizing the food photo taken by the phone camera and filling the corresponding nutritional information.

\section{Motivations}

People pay attention to fitness more nowadays, so proper diet became more important as well. However, delicious gourmet and easy access challenges people's ability to keep to their diet. Although people have many ways of logging their food and calories [8], it can still be difficult for people to keep a consistent log without resolution [9]. It can still be complicated for many people to keep track of their diet despite many food logging applications [10]. 


\subsection{Challenge 1}

It is easy for people to eat unrestrictedly, therefore, difficult to keep to a healthy diet. This is challenging from both the psychological and physical perspectives [11]. Even though there are various products that claim the effectiveness of controlling the diet, the actual effectiveness is still unknown.

\subsection{Challenge 2}

Results from having a good diet are subtle and delayed. Thus, following a diet might seem futile, and may discourage people from continuing their diet. Research shows that more and more people start to understand the importance of controlling diet, but very few are able to keep the healthy diet consistently. This is also an important reason why most people fail on controlling diet or weight.

\subsection{Challenge 3}

People lack the habit of logging their diet, so it is important to have a simple way for people to track their diet. Controlling diet requires persistence and motivation. While it is difficult to see the subtle changes, if people could monitor the steps and efforts clearly, it can provide another effective way of gaining confidence and motivation.

\section{SOLUTIONS}

Figure 1 shows an overview of the system. The system uses Tensorflow to train food images. TensorFlow is a multipurpose machine learning framework. TensorFlow can be used anywhere from training huge models across clusters in the cloud, to running models locally on an embedded system like your phone. In our application, the trained Tensorflow model will be embedded into the phone, so the performance can be optimized and guaranteed. On the other hand, a mobile application has been developed using Andriod Studio, which integrates the trained deep learning model and makes predictions to classify the food photos.

In addition, the mobile application allows users to log the food and calories and be able to see the history of the food, which is an essential component to keep users engaged and motivated.
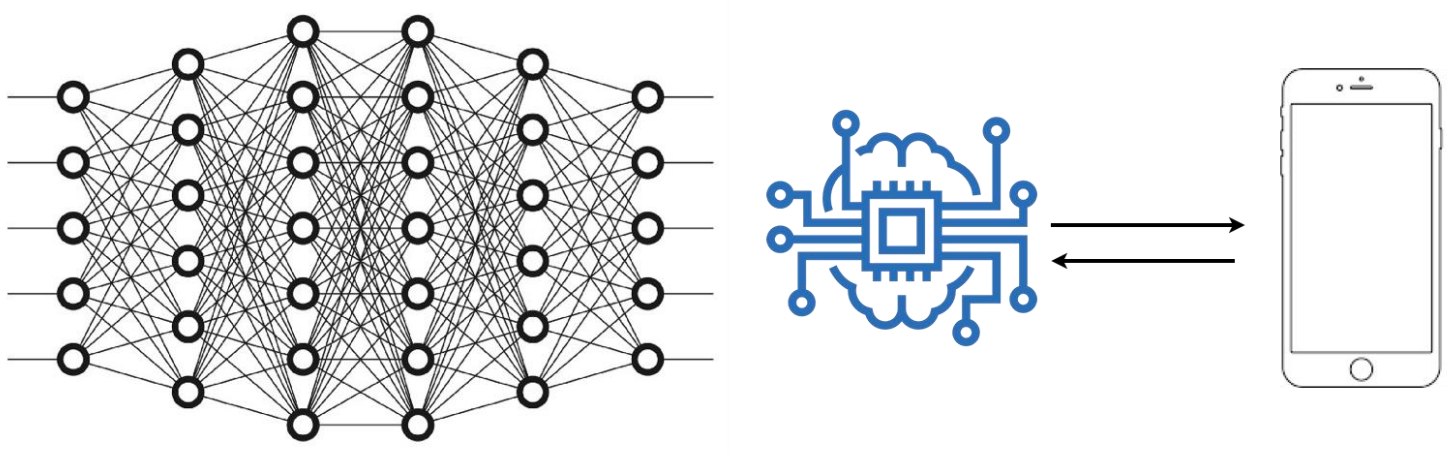

Figure 1. An Overview of the System 


\subsection{Addressing Challenge 1}

Provide food $\log$ for users to keep track of their calories and eating habits and give suggestions based on the current diet status. Build the food log utilizing the paper API to make the list able to take in new entries. The suggestions system uses the if/else logic to provide advice based on the user's dieting status.

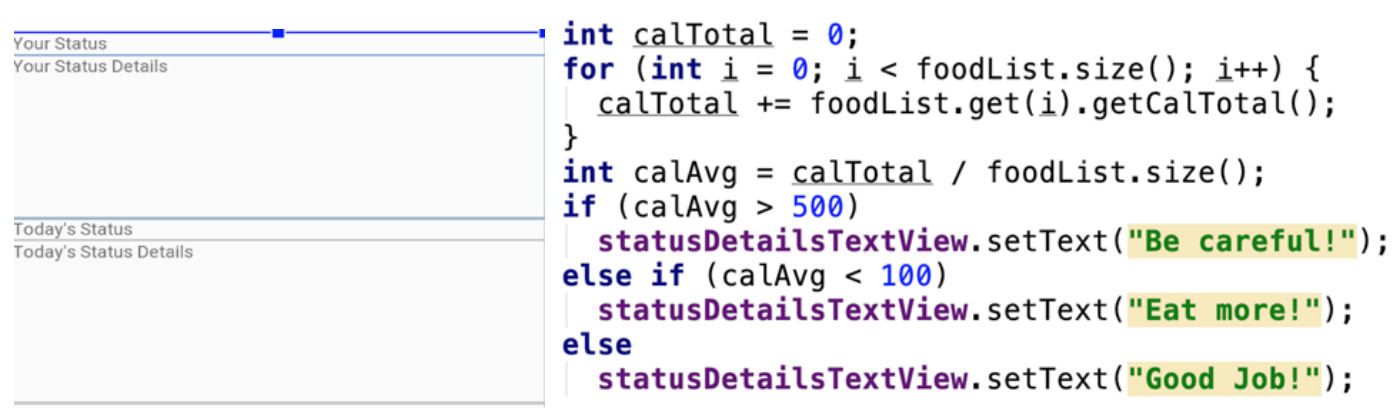

Figure 2. The UI of the Home Status Details Screen and A Code Excerpt

\subsection{Addressing Challenge 2}

By keeping track of eating habits, users can see their progression better and continue further with their diet. The continuous list of the food log can show the users their historical eating habits and compare them to those currently.

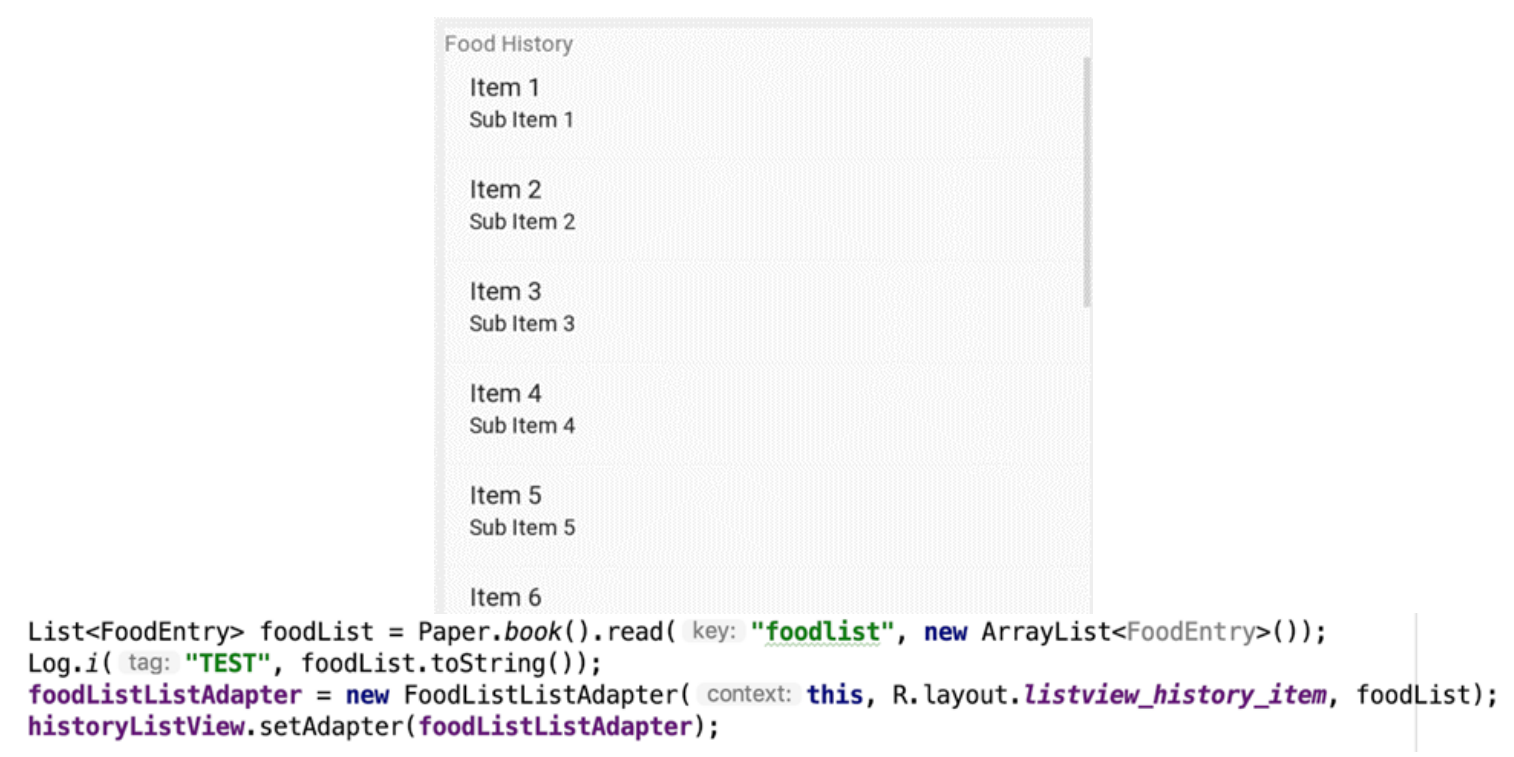

Figure 3. The UI of the Home History Screen and A Code Excerpt

\subsection{Addressing Challenge 3}

The simplicity of the food logging can help users build a habit of keeping track of their diet. By incorporating the image classification model, inception, the application is able to recognize the users' food through the camera. This feature allows the users to easily record their meals from photos taken of their food and develop a habit of logging their food through this simplicity. 


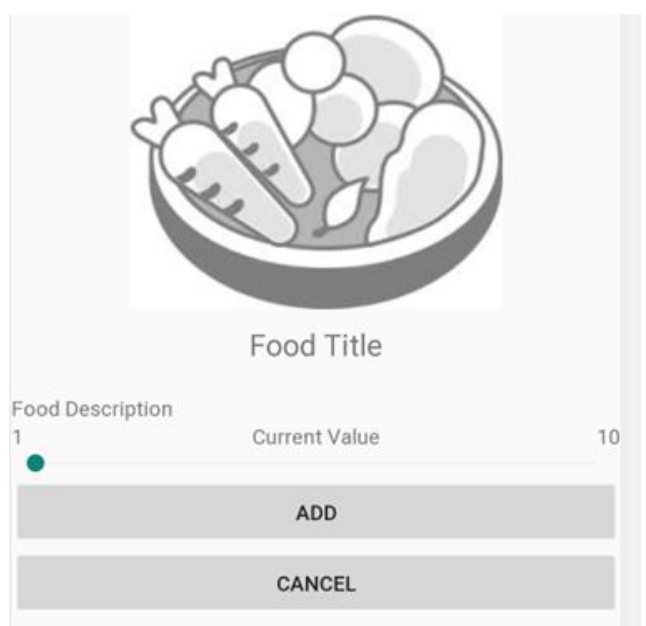

Figure 4. The UI of the Adding Food Screen

\section{EMPIRICAL RESULTS}

For the food classification feature to be accurate on user taken photos, which are usually of low quality and noisy lighting, the image classification model training data set has to be carefully chosen in order to yield the best performance on user photos [12]. In addition to choosing apt training images, the number of classification classes and the amount of images to train one class also heavily influences the accuracy of the model. From several trials of different model training, we found that when the amount of images in each class increased the test accuracy decreased, and when the amount of classes increased, the accuracy also decreased. This observation on training doesn't support the goal of the model since the model needs to incorporate many classes in order to recognize a wide variety of food, but if more classes are added to the training, the accuracy will decrease. Thus, it is important to train with more epochs supported by the observation that the accuracy increases as the number of training epochs increased.

\section{Accuracy with different amount of images \\ - 500 epochs -4000 epochs}

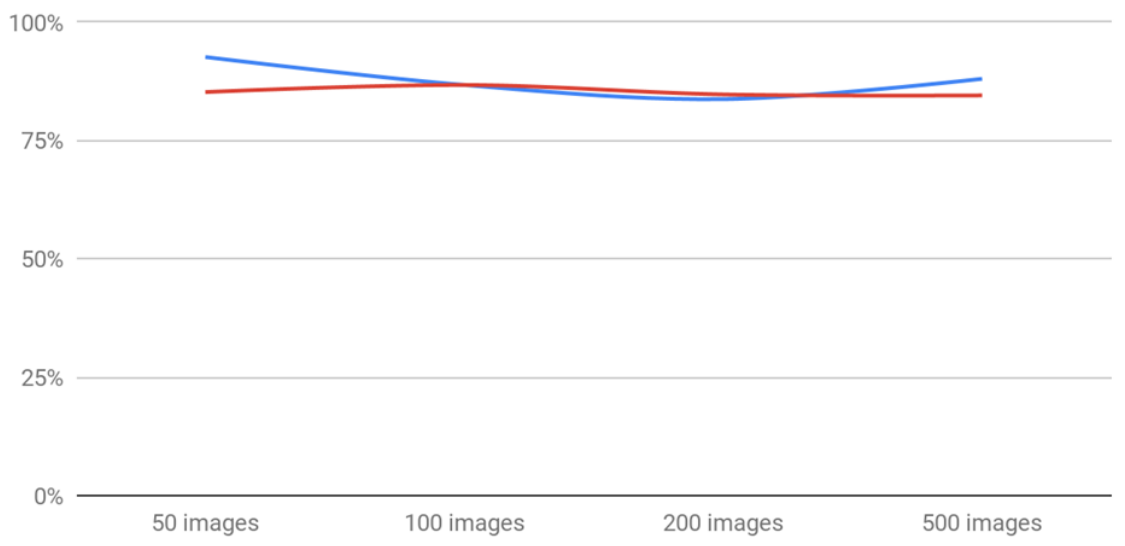

Figure 5. The Accuracy of Experiment 1 


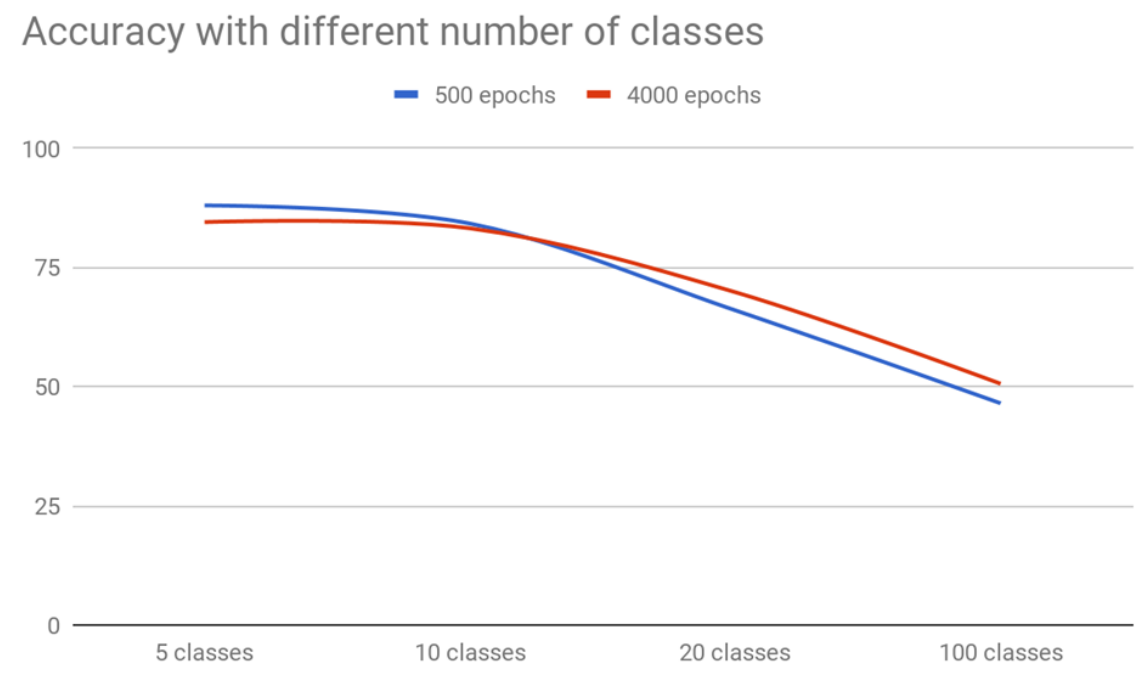

Figure 6. The Accuracy of Experiment 2

\section{RELATED WORK}

Related works solve some problems such as inaccuracy, volume of food, and inability to recognize all food in an image [13]. Manika Puri, Zhiwei Zhu, Qian Yu, Ajay Divakaran, and Harpreet Sawhney [14] found a solution to old food intake assessment that suffer from inaccuracy or complex lab measurements. Their solution is to use a mobile phone to capture images of foods, recognize food types, estimate their respective volumes and finally return quantitative nutritional information. Yuji Matsuda, Hajime Hoashi, and Keiji Yanai [15] proposed a two-step method to recognize all the food in multiple-food images. Unlike these related works, we propose to apply these methods into developing a solution that helps people log their food and calories and ultimately helping them form a habit of recording their meals.

\section{CONClusions ANd Future Work}

By incorporating a machine learning image classification model with the mobile app, we propose a way to simplify the process of recording everyday calories and eating habits. The solution to the difficulties that stop people from keeping a consistent diet plan can be solved by using the food recognition feature to identify the specific food and its nutritional information from user taken photos of their food either to post on social media or just for a personal record. In order to accommodate to various difficulties faced with extracting data from user photos, methods proposed by other computer vision researchers can be utilized to extract volume information from a food photo and to extract multiple food items' information from a single photo.

\section{REFERENCES}

[1] Rao, M., Afshin, A., Singh, G., \& Mozaffarian, D. (2013). Do healthier foods and diet patterns cost more than less healthy options? A systematic review and meta-analysis. BMJ open, 3(12), e004277.

[2] Piernas, C., Tate, D. F., Wang, X., \& Popkin, B. M. (2013). Does diet-beverage intake affect dietary consumption patterns? Results from the Choose Healthy Options Consciously Everyday (CHOICE) randomized clinical trial. The American journal of clinical nutrition, 97(3), 604-611. 
[3] Hawkes, C., Blouin, C., Henson, S., Drager, N., \& Dubé, L. (2009). Trade, food, diet and health: perspectives and policy options. John Wiley \& Sons.

[4] Albert, N. M., Butler, R., \& Sorrell, J. (2014). Factors related to healthy diet and physical activity in hospital-based clinical nurses. OJIN: The Online Journal of Issues in Nursing, 19(3).

[5] Coughlin, S. S., Whitehead, M., Sheats, J. Q., Mastromonico, J., Hardy, D., \& Smith, S. A. (2015). Smartphone applications for promoting healthy diet and nutrition: a literature review. Jacobs journal of food and nutrition, 2(3), 021

[6] Azar, K. M., Lesser, L. I., Laing, B. Y., Stephens, J., Aurora, M. S., Burke, L. E., \& Palaniappan, L. P. (2013). Mobile applications for weight management: theory-based content analysis. American journal of preventive medicine, 45(5), 583-589.

[7] El-Gayar, O., Timsina, P., Nawar, N., \& Eid, W. (2013). Mobile applications for diabetes selfmanagement: status and potential. Journal of diabetes science and technology, 7(1), 247-262.

[8] Hawkes, C., Jewell, J., \& Allen, K. (2013). A food policy package for healthy diets and the prevention of obesity and diet-related non-communicable diseases: the NOURISHING framework. Obesity reviews, 14, 159-168.

[9] Jacobs, D. R., \& Tapsell, L. C. (2013). Food synergy: the key to a healthy diet. Proceedings of the Nutrition Society, 72(2), 200-206.

[10] Sallis, J. F., Pinski, R. B., Grossman, R. M., Patterson, T. L., \& Nader, P. R. (1988). The development of self-efficacy scales for healthrelated diet and exercise behaviors. Health education research, 3(3), 283-292.

[11] Fraj, E., \& Martinez, E. (2006). Environmental values and lifestyles as determining factors of ecological consumer behaviour: an empirical analysis. Journal of Consumer Marketing, 23(3), 133144.

[12] Farinella, G. M., Allegra, D., \& Stanco, F. (2014, September). A benchmark dataset to study the representation of food images. In European Conference on Computer Vision (pp. 584-599). Springer, Cham.

[13] Kawano, Y., \& Yanai, K. (2013). Real-time mobile food recognition system. In Proceedings of the IEEE Conference on Computer Vision and Pattern Recognition Workshops (pp. 1-7).

[14] Puri, M., Zhu, Z., Yu, Q., Divakaran, A., \& Sawhney, H. (2009, December). Recognition and volume estimation of food intake using a mobile device. In 2009 Workshop on Applications of Computer Vision (WACV) (pp. 1-8). IEEE.

[15] Matsuda, Y., Hoashi, H., \& Yanai, K. (2012, July). Recognition of multiple-food images by detecting candidate regions. In 2012 IEEE International Conference on Multimedia and Expo (pp. 25-30). IEEE. 